\title{
Trends in Blood Stream Infections of Adolescents the Forthcoming Challenges in a North Indian Tertiary Care Hospital IGMC Shimla, India
}

\author{
Pankaj Katoch ${ }^{1 *}$ and Sanjay Chandel $^{2}$ \\ ${ }^{1}$ Senior Resident, Department of Microbiology, IGMC Shimla, India \\ ${ }^{2}$ Assistant Professor, Department of Forensic Medicine, DR. RKGMC Hamirpur, \\ Himachal Pradesh, India \\ *Corresponding Author: Pankaj Katoch, Senior Resident, Department of \\ Microbiology, IGMC Shimla, India.
}

Received: October 28, 2021

Published: November 24, 2021

(C) All rights are reserved by Pankaj Katoch and Sanjay Chandel.

\begin{abstract}
Background: Septicaemia and bacteremia are among the major challenges faced by health care professionals worldwide, these days leading to increased chances of morbidity and mortality for patients of all age groups.

The incidence among newborns can be low, but the chances of adverse consequences are increased. Blood culture forms a critical part of the evaluation of patients with suspected sepsis [1]. However, very little is known about the trends of bacterial bloodstream infections and antimicrobial susceptibilities among the adolescent population, as the literature is less. Hence, the present study is designed to evaluate the current trends in incidences and the etiological profile of BSI among adolescents using automated Bactec systems

Objective: To formulate guidelines for the care of adolescents from birth to 18 years of age.

Materials and Methods: All blood culture samples received in the Department of Microbiology for culture by Bactec Bd fx from July 2015 to June 2016 were included in the study. The blood culture was observed in the Bactec Bd fx system for at least 5 days before reporting as sterile.

Results: A total of 563 samples in the adolescent age group and suspected of BSI's received in the Department of Microbiology, IGMC, Shimla. All indoor and outdoor patients with temperatures $>=38$ degrees C. Among them, 324(57.5\%) were of males while $239(42.5 \%)$ were females. Among the total of 563 samples, 124(22\%) were found to be positive in culture, 426(75.7\%) were negative, while $13(2.3 \%)$ had contaminants defined by the growth of three different types of colonies in the medium. Among the 124 positive cases, 67(54\%) were men while 57(46\%) were women.

Conclusion: Bloodstream infection is an important cause of morbidity and mortality in patients of the adolescent age group. All febrile patients in the study group diagnosed with bacterial infections were included in this study. There was relatively high positivity in blood culture among the age group. Positivity was high in males compared to females.
\end{abstract}

Keywords: Infections; Culture; Bactec; Group of Adolescents

\section{Introduction}

Data especially from developed countries indicates that nearly 52 million incidents of sepsis occur throughout the world each year, with estimates of about six million deaths. The characteristic features of endothelial dysfunction by sepsis include an increase in the level of oxidative stress, systemic involvement, leading to inflammation, the glycocalyx degrades, leading to shedding, endothelial damage or death, the blood-tissue barrier is disrupted, more leukocyte adhesion occurs, consequently leading to the cascade of a procoagulant antifibrinolytic state [2].

Low- income and developing countries face a huge burden of bloodstream infections which in turn contribute to morbidity, mor- 
tality, and increased financial burden on patients or their families, as per Okeke., et al. [3] Reynolds R., et al. [4]. Diekema DJ., et al. [5]. Adolescent age group patients with BSI can present a dilemma in diagnosis and therapeutic difficulty because they can present with a normal or low body temperature as per Parajuli NP., et al. [6]. Bloodstream infections (BSIs) are among the main factors causing mortality and morbidity all over the world but are given less recognition. The terms bloodstream infection and bacteremia are concurrently in practice and point to the fact about the replication and growth of a microorganism in a culture from a patient with signs of infection. In practice, bacteremia can manifest from limiting infections to life-dangering septicemia which requires prompt antimicrobial treatment [7,8]. Staphylococcus aureus and Coagulase-negative staphylococci (CoNS) are some of the common Grampositive isolates. The virulence of CONS organisms is relatively lower, but they can cause significant infections in the bloodstream and other sites. Risk factors for these infections include the presence of invasive devices (such as catheters, central venous lines, intravascular catheters) and immunosuppressed states. It is very difficult to distinguish true infection from contamination. The true bacteremia of Staphylococcus aureus is relatively severe and has significant complications even with appropriate therapy. Streptococcus pyogenes is a common cause of pharyngitis and other associated invasive complications. When we consider Gram-negative bacteria, they are generally associated with hospital-acquired infections and catheter-related invasive devices. Pseudomonas aeruginosa is one of the most commonly implicated gram-negative aerobic bacteria in the differential diagnosis of hospital-acquired infections. Pseudomonas aeruginosa is one of the most commonly considered gramnegative aerobic bacilli in the differential diagnosis of several probable gram-negative infections, especially in the immunosuppressed hosts, is antibiotic-resistant, complicating therapy selection, and has a significant mortality rate. E.coli meningitis early can present without signs of nervous system involvement. Some other Gramnegative bacilli, such as Citrobacter koseri, Salmonella species, can cause brain abscesses in infants with meningitis. Salmonellae are motile Gram-negative bacilli that can infect a wide range of mammalian hosts. They cause several infections in humans including gastroenteritis, enteric fever, endovascular infection, focal or localized infections such as osteomyelitis or abscess.

Bloodstream infections leading to septicemia, are a big challenge in therapeutic medicine. This life-threatening infection gets worsened quickly due to the circulation of infecting pathogens or their toxic metabolites in the blood. Neonates and young children are among the most susceptible groups to catching diseases due to their weak immune status. The problem is more serious particularly in infants and young children with high-grade fever and undetected focus of infection [9]. Changing patterns of isolates, increasing rates of antimicrobial resistance, and the wide application of new medical technologies as the rampant use of dwelling devices can change the epidemiology and outcome of BSIs [10].

Therefore, it is necessary to continually review and update the epidemiology of BSI s mainly due to the antibiotic susceptibility pattern of circulating common pathogens, so that prompt and necessary empirical treatment of patients can be initiated [11].

Bloodstream infections (BSIs) are significant causes of morbidity and mortality worldwide but are poorly documented and do not receive the proper attention. Illness associated with bacteremia ranges from self-limiting infection to life-threatening sepsis, that is, mortality ranging from $20 \%$ to $50 \%$ [12].

BSIs are associated with an increased duration of hospitalization and are a huge financial burden on patients or their families. Improvement in clinical microbiology infrastructure and services for the empirical treatment guidelines could contribute to a better outcome [13].

Most studies related to the laboratory diagnosis of BSI focus either on infants or the adult population. This study in IGMC Shimla INDIA was conducted on the adolescent age group using an automated Bactec BD FX machine for culture. Therefore, the present study is being done to evaluate the incidence of BSIs among the adolescent age group.

\section{Objectives}

Determine the epidemiological BSIs among the adolescent age group using automated Bactec systems.

\section{Material and Methods}

- Study Design: Prospective observational study

- Study Setting: Department of Microbiology, Indira Gandhi Medical College And Hospital, Shimla, India

- $\quad$ Study Period: One year from July 2015 to June 2016.

\section{Inclusion criteria}

- All blood culture samples received in the Department of Microbiology for blood culture that falls within the age range of the study group. 
- $\quad$ Patient attendants were present with informed consent and were willing to study.

- $\quad$ Blood cultures from age groups.

\section{Exclusion criteria}

- Patient or their attendants not willing to study.

- Blood cultures showing mixed microbial growth.

\section{Methodology}

All blood culture samples of the age groups in the Department of Microbiology for culture were included in the study. The culture was observed in the Bactec bd fx system for at least 5 days before being reported as sterile.

The sample to be tested is inoculated into the vial for children, which is then inserted into the Bd Bactec fluorescent series instrument for incubation. Each bottle has a sensor that detects the increase in $\mathrm{CO}_{2}$ produced by the growth of microorganisms. The sensor monitors every 10 minutes for an increase in fluorescence, which is proportional to the amount of $\mathrm{CO}_{2}$ present. A positive reading indicates the tentative presence of viable pathogens in the bottle. The positive bottle will be sub-cultured on blood agar and McConkey agar plates. Following the subculture in solid media from each positive bottle, a smear will be prepared for gram staining from that blood culture vial. The smear will be analyzed for the presence of pathogens and the presumptive report will be transmitted to the respective departments. Escherichia coli ATCC 25922, Staphylococcus aureus ATCC25923, Staphylococcus epidermidis ATCC 12228, Streptococcus pneumonia ATCC 49619, and Pseudomonas aeruginosa ATCC 27853 were inoculated along with the test organisms. All positive isolates were stocked.

\section{Antibiotic susceptibility test}

The antibiotic sensitivity test was performed by diffusion of discs as per the CLSI guidelines. The antimicrobial susceptibility pattern was analyzed by the Kirby-Bauer disk diffusion method. The susceptibility of isolated organisms to agents was tested using Mueller-Hinton agar. Drug-resistant strains were evaluated to determine methicillin resistance in Staphylococcus aureus (MRSA) strains and extended-spectrum beta-lactamases (ESBLs) in gramnegative bacterial isolates.

\section{Statistical analysis}

Data were analyzed by statistical analysis-epi info7. The data was collected and analyzed in a spreadsheet. Analysis in terms of demography, positivity in samples, type of species, was done using statistical software epi-info version (7.1.1.0).

\section{Results}

A total of 563 samples from the adolescent group suspected of BSIs were received in the Department of Microbiology, IGMC, Shimla. Among them, 324(57.5\%) were male while 239(42.5\%) were of females (Figure 1).

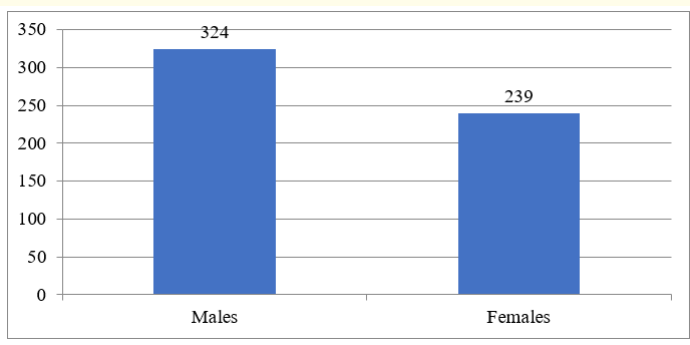

Figure 1: Distribution age group cases.

Among the total of 563 samples, 124(22\%) were positive in culture, $426(75.7 \%)$ were negative, while $13(2.3 \%)$ had contaminants (Figure 2).

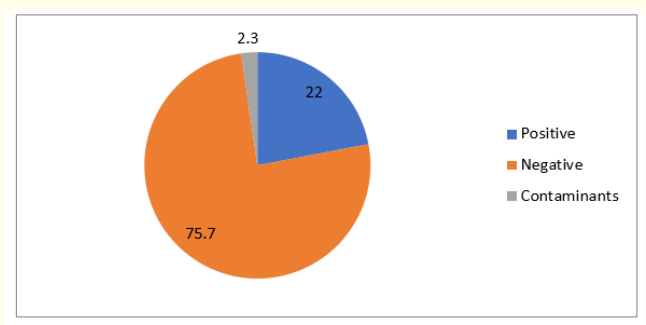

Figure 2: Culture results from the Adolescent age group.

Among the 124 positive cases, 67 (54\%) were men while $57(46 \%)$ were women (Table 1).

\begin{tabular}{|l|c|c|c|c|c|c|}
\hline \multirow{2}{*}{$\begin{array}{l}\text { Positive } \\
\text { Negative }\end{array}$} & \multicolumn{3}{|c|}{ Culture Results } & \multirow{2}{*}{ Total } \\
\cline { 4 - 7 } & \multirow{2}{*}{\begin{tabular}{l} 
Male \\
\multirow{3}{*}{ Gender }
\end{tabular}} & Contaminants & & & \\
\cline { 3 - 7 } & \multirow{2}{*}{ Female } & 67 & 249 & 8 & 324 \\
\cline { 3 - 7 } & & Count & 57 & 177 & 5 & 239 \\
\cline { 3 - 7 } & & $46.0 \%$ & $41.5 \%$ & $38.5 \%$ & $42.5 \%$ \\
\hline $\begin{array}{l}\text { Total } \\
\%\end{array}$ & Count & 124 & 426 & 13 & 563 \\
\hline
\end{tabular}

Table 1: Gender distribution of culture-positive and negative cases. 


\begin{tabular}{|l|c|}
\hline S. aureus & $18(14.5 \%)$ \\
\hline Group B Streptococcus & $14(11.29 \%)$ \\
\hline $\begin{array}{l}\text { Coagulase-negative Staphylococ- } \\
\text { cus species }\end{array}$ & $8(6.45 \%)$ \\
\hline
\end{tabular}

Table 2: Gram-Positive isolates.

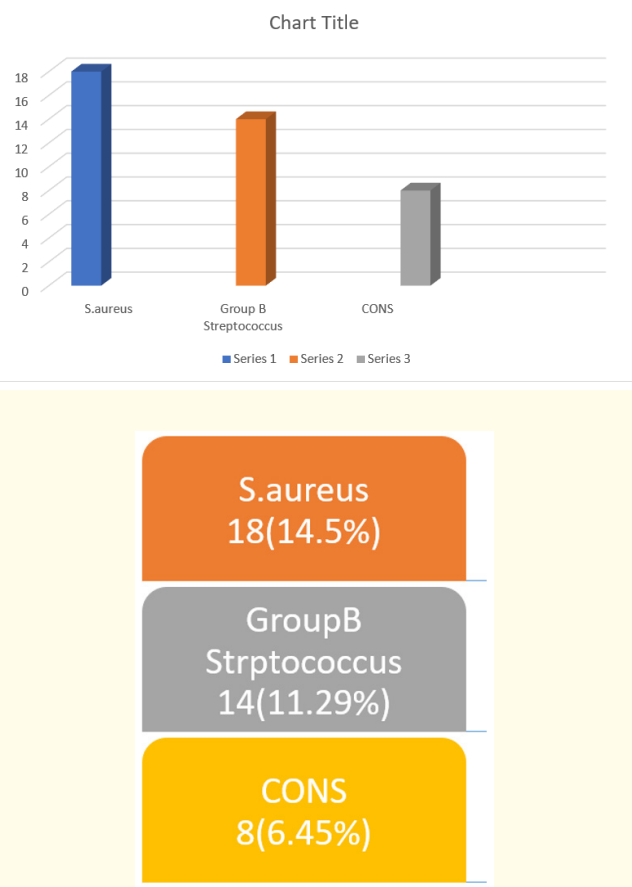

Figure a

\begin{tabular}{|l|c|}
\hline E. coli & $24(19.35 \%)$ \\
\hline K. pneumoniae & $20(16.13 \%)$ \\
\hline P. aeruginosa & $18(14.52 \%)$ \\
\hline Salmonella Typhi & $12(9.68 \%)$ \\
\hline Citrobacter koseri & $5(4.03 \%)$ \\
\hline Acinetobacter baumanni & $5(4.03 \%)$ \\
\hline
\end{tabular}

Table 3: Gram-negative Isolates.

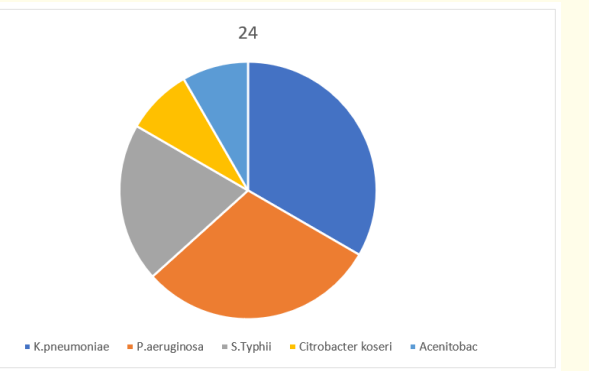

Figure b: Gram-Negative Isolates.

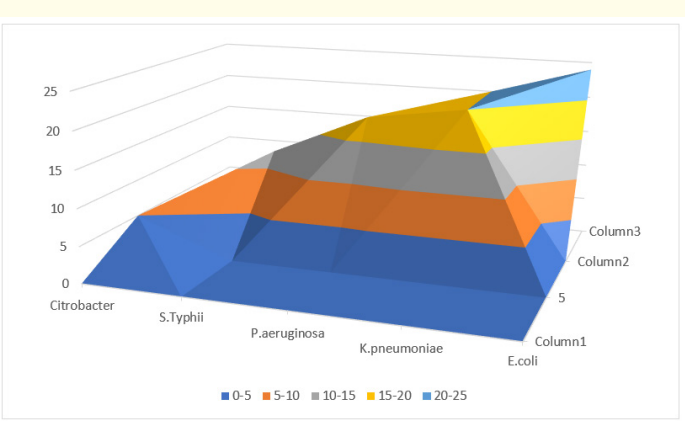

Figure c: Gram-Negative isolates in ascending order.

Of the 40 Gram-positive isolates, S. aureus was isolated in 18 cases (14.5\%), Group B Streptococcus 14(11.29\%), Coagulase-negative Staphylococcus species in $8(6.45 \%)$ cases. Regarding gramnegative isolates, E. coli was found in $24(19.35 \%)$ cases of $K$. pneumoniae 20(16.13\%) cases, P. aeruginosa 18 (14.52\%), Salmonella Typhi 12(9.68\%) Citrobacter koseri 5(4.03\%) and Acinetobacter baumanni 5(4.03\%) cases. Extended beta spectrum isolates were reported to be 20 of 84 (23\%) and methicillin-resistant Staphylococcus aureus cases 5 of 40 bacteria that is 12.5 (\%).

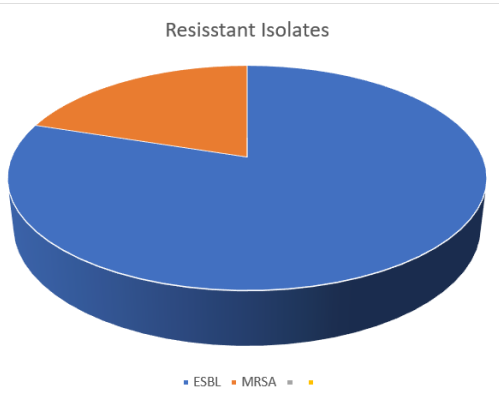

Figure d

\section{Discussion}

Based on the present study, we attempted to determine epidemiologic BSIs among the adolescent age groups. Detecting bacteria in the blood has an important role in the diagnosis of a febrile patient; to establish the cause of infection, to assure the treating physician about the therapy chosen, and to provide up-to-date information on the local etiologic patterns and antibiotic sensitivities, as this will guide the clinician in the management of the patient.

The characteristic features of endothelial dysfunction by sepsis are an increase in the level of oxidative stress, systemic involvement, and subsequent inflammation, the glycocalyx degrades, leading to shedding, endothelial damage, or death, the blood-tissue 
barrier is disrupted, more leukocyte adhesion occurs, consequently resulting in a cascade of procoagulant and anti-fibrinolytic state $[2]$.

Rapid detection and isolation of clinically important microorganisms in blood cultures are essential to determine antimicrobial susceptibility patterns for prompt administration of antimicrobial therapy has been demonstrated to reduce effectively the morbidity and mortality with bloodstream infections. Therefore, blood culture remains the mainstay of the investigation of definitive diagnosis and management of bloodstream infection in the adolescent age group, despite recent advances in the molecular diagnosis of bacterial sepsis [14]. Blood cultures are usually obtained along with clinical investigations to search for a focus of infection and therefore provide specific antimicrobial therapy.

In the present study, a total of 563 samples in the adolescent age group suspected of BSI's were received at the Department of Microbiology, IGMC, Shimla. Among them, 324(57.5\%) were men while 239(42.5\%) were women. Among the total of 563 samples, $124(22 \%)$ were found positive in culture, $426(75.7 \%)$ negative while $13(2.3 \%)$ were having contaminants that grow more than three types of colonies in the medium. Among the 124 positive cases, 67(54\%) were men while 57(46\%) were women.

The culture Positivity rates documented in other studies conducted similarly to our study settings reported the following results 48.9\% Meremikwu MM., et al. [13], 20.2\% Arora U and Devi P [14], 18.7\% Murthy DS and Gyaneshwari M [15] and 16.6\% Qureshi and Aziz F [16] and our results are in agreement with other studies conducted before.

This variation might be because patients admitted to the tertiary care hospital received antibiotics before they came to the tertiary care hospital and another factor contributing in our country India is that in most cases self-medication is very common as the medicines are available at the counter. It is implied in a tertiary level institution that patients have received prior antibiotics and not responding they present themselves in a tertiary care institution. Thus variations in culture positively rate are most probably due to prior treatment with antibiotics $[17,18]$.

Bloodstream infections continue to be a challenge for infectious disease physicians due to the ever-changing bacterial etiology and the emergence of antimicrobial resistance. Early detection of causative pathogens and determination of their susceptibility profile is necessary to help clinicians decide on rational empirical therapy, which decreases the emergence of resistance. Despite advances in diagnosis and treatment, bacterial sepsis continues to be a major cause of adolescent morbidity and mortality, particularly in developing countries. The causative agents of sepsis and their antibiotic susceptibility patterns also become varied from time to time and from place to place. The findings of a study conducted also emphasize that the duration of hospital stay and mechanical ventilation of children (neonates, infants, and children 1 to 18 yrs of age) admitted to NICUs and PICUs have a significant association with ventilator-associated pneumonia which in turn, leads to an increase in the mortality rate [19]. Detection of bacteria in the blood has a very important role in the diagnosis of a patient with BSI especially in Pyrexia of Unknown origin infection, assuring the clinician about the therapy chosen, and provides valuable insight on local etiologic patterns and sensitivities as this will help the clinician in the treatment of the patient.

\section{Conclusion}

This study provides some insight into local trends and bacterial etiology of bloodstream infections among patients in the adolescent age group. There was relatively high positivity in blood culture among the adolescent group. Positivity was high in males compared to females. The exact contributing factors for bloodstream infections (BSI) within these groups need to be further elucidated. Rational use of antibiotics, formulation of antibiotic policy, and prompt therapy of bloodstream infections for the effective management and prevention of drug resistance are urgently needed in setting, as noted by the prevalence of extended-spectrum beta-lactamases and Methicillin-resistant Isolates increasing prevalence.

\section{Consent for Publication}

Not applicable.

\section{Availability of Data and Materials}

Not applicable

\section{Competing Interests}

Nil.

\section{Funding}

Nil.

\section{Acknowledgements}

Nil everything from own pocket. 


\section{Bibliography}

1. Katoch Pankaj. "Incidence and Etiological Profile of Gram-Positive Bacterial Positivity in Invasive Bloodstream Infections in All Age Groups Using Automated Bactec in Tertiary Care Hospital of North India". International Academic Journal of Applied Bio-Medical Sciences 2.4 (2021).

2. Marta Martín-Fernandez., et al. "Shared Features of Endothelial Dysfunction between Sepsis and Its Preceding Risk Factors (Aging and Chronic Diseases)". Journal of Clinical Medicine 7.11 (2018): 400.

3. Okeke IN., et al. "Antimicrobial resistance in developing countries. Part II: strategies for containment". The Lancet Infectious Diseases 5 (2005): 568-580.

4. Reynolds R., et al. "Antimicrobial susceptibility of the pathogens of bacteremia in the UK and Ireland 2001-2002: the BSAC bacteremia resistance surveillance program". Journal of Antimicrobial Chemotherapy 53 (2004): 1018-1032.

5. Diekema DJ., et al. "Epidemiology and outcome of nosocomial and community-onset bloodstream infection". Journal of Clinical Microbiology 41 (2003): 3655-3660.

6. Parajuli NP., et al. "Evaluating the Trends of Bloodstream Infections among Pediatric and Adult Patients at a Teaching Hospital of Kathmandu, Nepal: Role of Drug-Resistant Pathogens". Canadian Journal of Infectious Diseases and Medical Microbiology (2017): 8763135.

7. Larry B., et al. "Bloodstream Infections in Hospitalized Children: Epidemiology and Antimicrobial Susceptibilities". The Pediatric Infectious Disease Journal 35.5 (2016): 507-510.

8. Surbhi Leekha., et al. "General Principles of Antimicrobial Therapy". Mayo Clinic Proceedings 86.2 (2011): 156-167.

9. Seifert $\mathrm{H}$ and Wisplinghoff H. "Bacteriology General". In: Borriello SP, Murray RP, Funke G editors. Topley, and Wilsons Microbiology and Microbiological infections. $10^{\text {th }}$ ed 1 (2001): 509-516.

10. Young LS. "Sepsis syndrome". In: Mandell GL, Mandell JE, Benett JE, Dolin R editors. Principles and practice of infectious diseases, 5thed Churchill Living stone New York (2005): 806-819.

11. Wattal C., et al. "Ecology of bloodstream infection and antibiotic resistance in intensive care unit at a tertiary care hospital in North India". The Brazilian Journal of Infectious Diseases 18.3 (2014): 245-251.
12. Malik Shakir Hussain., et al. "Bloodstream infections in the pediatric population in a tertiary care hospital". International Journal of Contemporary Medical Research 6.2 (2019): B10B12.

13. Meremikwu MM. "Bacterial isolates from blood cultures of children with suspected septicemia in Calabar, Nigeria". BMC Infectious Diseases 5 (2005): 110.

14. Arora U and Devi P. "Bacterial profile of bloodstream infections and antibiotic resistance pattern of isolates". JK Science 9 (2007): 186-190.

15. Murthy DS., et al. "Blood cultures in pediatric patients: a study of clinical impact”. Indian Journal of Medical Microbiology 25 (2007): 220-224.

16. Qureshi M and Aziz F. "Prevalence of microbial isolates in blood cultures and their antimicrobial susceptibility profiles". Biomedica 27 (2011): 136-139.

17. Pankaj Katoch. "Bacteriological Profile and Antibiotic Sensitivity Pattern in Early Onset Sepsis and Late-Onset Sepsis and comparison of ampicillin gentamicin combination with regard to $3^{\text {rd }}$ generation cephalosporins for empirical treatment initiation". International Academic Journal of Applied Bio-Medical Sciences 2.4 (2021): 12-16.

18. Donkor ES., et al. "Self-medication practices with antibiotics among tertiary level students in Accra, Ghana: a cross-sectional study". International Journal of Environmental Research and Public Health 9.10 (2010): 3519-3529.

19. Singh Surinder., et al. "Mortality and Morbidity among Children Diagnosed with Ventilator-Associated Pneumonia in Tertiary Care Hospital". International Academic Research Journal of Internal Medicine and Public Health 2.4 (2021).

\section{Volume 4 Issue 12 December 2021 (C) All rights are reserved by Pankaj Katoch and Sanjay Chandel.}

